ITP-Budapest Report 648

\title{
6-loop anomalous dimension of a single impurity operator from AdS/CFT and multiple zeta values
}

\author{
Zoltán Bajnok ${ }^{a}$ and Omar el Deeb ${ }^{b}$ \\ December 22, 2018 \\ ${ }^{a}$ Theoretical Physics Research Group of the Hungarian Academy of Sciences, \\ ${ }^{b}$ Institute for Theoretical Physics, Roland Eötvös University, \\ H-1117 Pázmány s. 1/A, Budapest, Hungary
}

\begin{abstract}
Anomalous dimension of the simplest nontrivial single impurity operator in the $\beta=\frac{1}{2}$ deformed theory is determined at six loops from the AdS/CFT correspondence. Lüscher correction is evaluated at next-to-next-to-leading order (NNLO) in terms of multiple zeta values. The result can be simplified into the products of simple zeta functions and the same form of the correction is expected for the Konishi operator at six loops, too.
\end{abstract}

\section{Introduction}

AdS/CFT correspondence [1] relates the string energies on the $A d S_{5} \times S^{5}$ background to the anomalous dimensions of gauge invariant operators in maximally supersymmetric four dimensional $S U(N)$ gauge theory. The correspondence is particularly useful in the large $N$ limit when it can be described by a two dimensional integrable field theory, see [2] for a general introduction and references therein. The advantage of the integrable two dimensional point of view lies in its non-perturbative nature, which nevertheless, can be used to calculate perturbative quantities, too. Indeed, the leading finite size correction of a two particle state can be described in terms of the asymptotical Bethe ansatz and the generalized Lüscher formulas [3, which, when expanded in the coupling constant, provides the exact perturbative anomalous dimension of the Konishi operator up to seven loops. The direct perturbative gauge theory calculations are very cumbersome and have been evaluated for the Konishi operator up to 4 loops [4, 5] only, where the wrapping part of the correction matches exactly the Lüscher type finite size correction [3]. Thus the integrability based "string theory" techniques provide a way to go beyond the available perturbative calculations and collect information about higher order results. The simplest situation in this respect is the calculation of the anomalous dimension of a single impurity operator. Such operator is trivial in the $\mathcal{N}=4 \mathrm{SYM}$ but has non-vanishing anomalous dimension in orbifold or $\beta$-deformed theories. The leading and next-to-leading wrapping correction have been calculated in [6] and in [7] and the aim of the present paper is to extend the calculation to next-to-next-to-leading order (NNLO). This order is important in the sense that it is not affected by double wrapping corrections thus the calculation based on the Lüscher formula provides and exact answer.

The $\beta$-deformed theory is an $\mathcal{N}=1$ supersymmetric, exactly marginal deformation of the $\mathcal{N}=4$ SYM and has been always the testing ground of the AdS/CFT duality. The dispersion relation of the excitations was calculated exactly in [8], which together with wrapping corrections [9, 10] provides the exact anomalous dimension of a single impurity operator $\operatorname{Tr}\left(X Z^{L}\right)$. On the string theory side this 
operator corresponds to a one particle state in the $s u(2)$ sector in finite volume. The leading finite size effect follows from the momentum quantization, or asymptotical Bethe Ansatz equation [11]. As the volume decreases Lüscher/wrapping type correction becomes important [3, 6, 12] and for an exact description they have to be summed up. An educated way to take all finite size correction into account would be to extend the Thermodynamic Bethe Ansatz of the $\mathcal{N}=4$ theory [13, 14, 15, 16, 17, 18, 19] to the $\beta$-deformed case. The first step into this direction is done: in [20] the authors extended the functional $Y$ system relations, while in [21] the scattering matrix of the theory together with its twisted boundary condition were identified.

We are interested in the $\beta$-deformed theory at a particular value of $\beta$, namely for the simplest nontrivial $\beta=\frac{1}{2}$ and evaluate the Lüscher correction at NNLO. This value of $\beta$ is interesting as it correspond to a particular orbifold theory, too. As it was conjectured in 22 the anomalous dimension of the $s u(2)$ particle $\left(\operatorname{Tr}\left(X Z^{3}\right)\right)$ in the $\beta=\frac{1}{2}$-deformed theory coincides with the anomalous dimension of the $s l(2)$ particle $(\operatorname{Tr}(D Z Z))$ of the orbifold theory. A clear description of the relation can be found in [23] where a TBA equation was also proposed to describe the single impurity excitation of the orbifold theory. The proposed infinite coupled integral equations in principle exactly describe the energy of the one particle state for any volume and coupling. However, as it was shown in [23], following the work of [24, 25, 26], it reduces in the weak coupling limit to the Lüscher correction and that is what we are going to evaluate now. We will focus on the structure of the result, especially how the various terms of the perturbation theory appear in terms of multiple zeta values.

Multiple zeta values (MZVs) are the generalization of zeta functions:

$$
\zeta\left(a_{1}, a_{2}, \ldots, a_{n}\right)=\sum_{j_{1}>j_{2}>\ldots>j_{n}>0} \frac{1}{j_{1}^{a_{1}} j_{2}^{a_{2}} \ldots j_{n}^{a_{n}}}
$$

and are trivially related to the values of nested harmonic sums taken at infinity. They appear at various places of physics and mathematics including number theory, combinatorics, quantum field theory, statistical physics. For a recent review see [27] and references therein. What we would like to describe here is how the various MZVs appear at a given order of the calculation. We will see that at leading order we have simple zeta function, the next-to-leading order double MZVs will appear, while the novel result shows the appearance of triple MZVs at NNLO. Not only the depth of the MZVs increases with the orders but also their transcendentality degree. There are several nontrivial relations among the MZVs of a given transcendentality degree and one can always choose a good basis [28. Any basis above transcendentality 8 contain MZVs which are irreducible in the sense that cannot be written as products of simple zeta functions. As the anomalous dimension of the simplest nontrivial operator at NLO had transcendentality 7 such irreducible MZVs did not appear [7]. At the NNLO the transcendentality is 9 so we expect the appearance of irreducible MZVs. Let us anticipate our result.

We calculate the anomalous dimension of the operator $\operatorname{Tr}(X Z)$ in the $\beta=\frac{1}{2}$ deformed theory, or equivalently the anomalous dimension of the $\operatorname{Tr}(D Z Z)$ operator in the $\mathbb{Z}_{2}$ orbifold theory. On the string theory side they correspond to the energy of a one particle state in finite volume $L=1$. It is a very special state as it has a vanishing rapidity $u=0,(p=\pi)$, which is not effected by finite size corrections. In contrast, the energy is shifted by vacuum polarization effects as

$$
E=E_{A B A}+\Delta E
$$

The asymptotical Bethe Ansatz energy $E_{A B A}$ is simply the dispersion relation of a standing particle:

$$
\begin{aligned}
E_{A B A} & =\sqrt{1+16 g^{2} \sin ^{2}\left(\frac{p}{2}\right)}=\sqrt{1+16 g^{2}} \\
& =1+8 g^{2}-32 g^{4}+256 g^{6}-2560 g^{8}+28672 g^{10}-344064 g^{12}+O\left(g^{13}\right)
\end{aligned}
$$

while $\Delta E$ corresponds to the wrapping interactions and has the expansion

$$
\Delta E=\Delta E_{4} g^{4}+\Delta E_{5} g^{10}+\Delta E_{6} g^{12}+\ldots
$$


In [6] the LO correction was calculated

$$
\Delta E_{4}=128(4 \zeta(3)-5 \zeta(5))
$$

The NLO correction turned out to be [7]:

$$
\Delta E_{5}=-128\left(12 \zeta(3)^{2}+32 \zeta(3)+40 \zeta(5)-105 \zeta(7)\right)
$$

In the main part of the paper we evaluate the NNLO correction and obtain

$$
\Delta E_{6}=-128\left(48 \zeta(3)^{2}-592 \zeta(5)-24 \zeta(3)(8+15 \zeta(5))-322 \zeta(7)+1701 \zeta(9)\right)
$$

which completes the anomalous dimension of the operator up to six loops. One can observe that the transcendentality is 9 and that no irreducible MZV appeared. This is due to an unexpected cancellation during the calculation. Let me note also, that the form of the correction matches exactly with other 6 loop results like the ones in [29, 23].

The rest of the paper is organized as follows: In the next Section we present the Lüscher correction for a one particle state in the $\beta=\frac{1}{2}$ deformed theory by recalling the available formulas from the literature. Section 3 contains the results of the calculation. As we used a novel method to sum up the various terms we present the four and five loop results in this fashion and show how we obtained the result at six loops. We pay particular attention how the various MZVs appear and describe how they can be simplified. Finally we conclude in section 4 and explain how our novel results could be used. The Appendix contains some technical information, especially on how we summed up nested harmonic sums.

\section{Calculation of the anomalous dimension from AdS/CFT}

As the anomalous dimension of the single impurity operator corresponds to a standing particle state, we have to calculate the standard Lüscher correction. It describes how the one particle energy (dispersion relation)

$$
E=E(p=\pi)=\sqrt{1+16 g^{2} \sin ^{2}\left(\frac{p}{2}\right)}=\sqrt{1+16 g^{2}}=\epsilon
$$

is modified due to vacuum polarization effects. The $p=\pi$ momentum corresponds to vanishing rapidity

$u=0$ which is protected by symmetry. Consequently the leading finite size correction is the energy correction which originates from virtual particles propagating around the circle and can be written as

$$
\Delta E=-\sum_{Q=1}^{\infty} \int \frac{d q}{2 \pi} \operatorname{sir}\left(S_{Q 1}^{Q 1}(q, 0)\right) e^{-\tilde{\epsilon}_{Q}(q) L}+O\left(g^{16}\right)
$$

Here we sum up for all bound-states of charge $Q$ of the mirror model, whose momenta are $q, S_{Q 1}^{Q 1}$ describes how they scatter on the fundamental particle and $\tilde{\epsilon}(q)$ denotes their mirror energy. We have to expand this expression in NNLO in $g$. The above form exactly describes the energy correction up to the order $g^{14}$ only since at the order $g^{16}$ double wrapping effects will contribute, too. Let us analyze the $g$ dependence of the various terms.

\section{Exponential factor}

The mirror energy has the following parametrization

$$
e^{-\tilde{\epsilon}_{Q}(q)}=\frac{z^{-}(q, Q)}{z^{+}(q, Q)} \quad ; \quad z^{ \pm}(q, Q)=\frac{q+i Q}{4 g}\left(\sqrt{1+\frac{16 g^{2}}{q^{2}+Q^{2}}} \pm 1\right)
$$




\section{Matrix part of the scattering matrix}

The contribution of the scattering matrix can be factored as

$$
\mathrm{s} \operatorname{Tr}\left(S_{Q 1}^{Q 1}(q, 0)\right)=S_{\text {scalar }}(q, 0) \mathrm{s} \operatorname{Tr}\left(S_{\text {matrix }}^{\text {su(2) }}(q, 0)\right)^{2}
$$

where the super-trace of the matrix part contain the contributions of all polarizations. The various polarizations of the mirror bound-states can be labeled in the super-space formalism as $\left(w_{3}^{j} w_{4}^{Q-j}\right.$, $\left.w_{3}^{j} w_{4}^{Q-2-j} \theta_{1} \theta_{2}, w_{3}^{j} w_{4}^{Q-1-j} \theta_{3}, w_{3}^{j} w_{4}^{Q-1-j} \theta_{4}\right)$ see [30, 3] for the details. The super-trace in the $\beta$ deformed theory evaluates in the previous basis as

$$
\operatorname{sTr}\left(S_{\text {matrix }}^{\text {su(2) }}(z, x)\right)=\sum_{j=0}^{Q} S B 1_{j}(z, x)+\sum_{j=0}^{Q-2} S B 2_{j}(z, x)+i \sum_{j=0}^{Q-1} S F 1_{j}(z, q)-i \sum_{j=0}^{Q-1} S F 2_{j}(z, q)
$$

The S-matrix elements can be extracted from [3]. In calculating the corrections for the $s u(2)$ representative neither of the S-matrix contributions depends on $j$ and they read explicitly as

$$
\begin{gathered}
S B 1_{j}(z, x)=\frac{z^{+}-x^{+}}{z^{-}-x^{+}} \frac{\tilde{\eta}_{1}}{\eta_{1}} \quad ; \quad S B 2_{j}(z, x)=\frac{z^{+}-x^{-}}{z^{-}-x^{+}} \frac{\left(1-x^{+} z^{-}\right)}{\left(1-x^{-} z^{-}\right)} \frac{x^{-}}{x^{+}} \frac{\tilde{\eta}_{1}}{\eta_{1}}\left(\frac{\tilde{\eta}_{2}}{\eta_{2}}\right)^{2} \\
S F 1_{j}(z, x)=\frac{z^{+}-x^{-}}{z^{-}-x^{+}} \frac{\tilde{\eta}_{1}}{\eta_{1}} \frac{\tilde{\eta}_{2}}{\eta_{2}} \quad ; \quad S F 2_{j}(z, x)=\frac{z^{+}-x^{+}}{z^{-}-x^{+}} \frac{\left(1-x^{+} z^{-}\right)}{\left(1-x^{-} z^{-}\right)} \frac{x^{-}}{x^{+}} \frac{\tilde{\eta}_{1}}{\eta_{1}} \frac{\tilde{\eta}_{2}}{\eta_{2}}
\end{gathered}
$$

The appearing string frame factors can be written as $\frac{\tilde{\eta}_{1}}{\eta_{1}}=\sqrt{\frac{z^{-}}{z^{+}}}$and $\left(\frac{\tilde{\eta}_{2}}{\eta_{2}}\right)^{2}=\frac{x^{-}}{x^{+}}$. The $x^{ \pm}$parameters depend on the momentum the usual way

$$
x^{ \pm}(p)=\frac{\cot \frac{p}{2} \pm i}{4 g}\left(1+\sqrt{1+16 g^{2} \sin ^{2} \frac{p}{2}}\right)
$$

which in our case results in

$$
x^{ \pm}= \pm x= \pm \frac{i}{4 g}(1+\epsilon) \quad ; \quad \frac{x^{-}}{x^{+}}=-1
$$

With these variables the super-trace of the matrix part takes a particularly simple form

$$
\operatorname{sTr}\left(S_{\text {matrix }}^{\text {su(2) }}(z, x)\right)=\frac{2 x\left(1+2 Q z^{-}\left(x-z^{+}\right)\right)}{\left(x-z^{-}\right)\left(1+x z^{-}\right)} e^{-\tilde{\epsilon}_{Q}(q) / 2}
$$

We have checked that the matrix part of the $s l(2)$ representative of the orbifold model gives the same result. There, the deformation is such that the fermions do not contribute as the two undeformed fermionic S matrix elements are the same [6, 31].

\section{Scalar part of the scattering matrix}

The scalar part of the scattering matrix of a charge $Q$ bound-state can be obtained by multiplying the scalar factors of its individual scattering constituents. The charge $Q$ bound-state composed of elementary magnons as $z=\left(z_{1}, \ldots, z_{Q}\right)$, such that $z^{-}=z_{1}^{-}$and $z_{Q}^{+}=z^{+}$and the bound-state condition is also satisfied $z_{i}^{+}=z_{i+1}^{-}$. Thus the full scalar factor as the product of the elementary scalar factors turns out to be [3]:

$$
S_{\text {scalar }}(z, x)=\prod_{i=1}^{Q} S^{s l(2)}\left(z_{i}, x\right)=\prod_{i=1}^{Q} e^{-2 i \sigma\left(z_{i}, x\right)} \frac{z_{i}^{-}-x^{+}}{z_{i}^{+}-x^{-}} \frac{1-\frac{1}{z_{i}^{+} x^{-}}}{1-\frac{1}{z_{i}^{-} x^{+}}}
$$


In calculating the Lüscher correction we have to evaluate this expression when $z$ is in the mirror kinematics $\left(\left|z^{-}\right|<1,\left|z^{+}\right|>1\right)$. The analytical continuation has been carefully elaborated in [32]:

$$
S_{\text {scalar }}^{-1}(z, x)=\Sigma_{Q, 1}^{2}(z, x) S^{s u(2)}(z, x)
$$

A particularly good feature of the formula is that both expressions depend on $z^{ \pm}$only and not on the individual $z_{i}^{ \pm}$. Explicitly the $s u(2)$ scalar factors read as:

$$
S^{s u(2)}(z, x)=\frac{\left(z^{+}-x^{-}\right)\left(z^{+}-x^{+}\right)}{\left(z^{-}-x^{+}\right)\left(z^{-}-x^{-}\right)} \frac{\left(1-\frac{1}{z^{+} x^{-}}\right)\left(1-\frac{1}{z^{+} x^{+}}\right)}{\left(1-\frac{1}{z^{-} x^{+}}\right)\left(1-\frac{1}{z^{-} x^{-}}\right)}
$$

while for the case $\left|x^{ \pm}\right|>1$ following [32] we can write

$$
\begin{aligned}
-i \log \Sigma_{Q, 1}(z, x)= & \Phi\left(z^{+}, x^{+}\right)-\Phi\left(z^{+}, x^{-}\right)-\Phi\left(z^{-}, x^{+}\right)+\Phi\left(z^{-}, x^{-}\right) \\
& +\frac{1}{2}\left[-\Psi\left(z^{+}, x^{+}\right)+\Psi\left(z^{+}, x^{-}\right)-\Psi\left(z^{-}, x^{+}\right)+\Psi\left(z^{-}, x^{-}\right)\right] \\
& +\frac{1}{2 i} \log \left[\frac{\left(z^{+}-x^{+}\right)\left(x^{-}-\frac{1}{z^{+}}\right)^{2}}{\left(z^{+}-x^{-}\right)\left(x^{-}-\frac{1}{z^{-}}\right)\left(x^{+}-\frac{1}{z^{-}}\right)}\right]
\end{aligned}
$$

We have to be careful as the conventions of [32] are different from ours. To turn into our conventions one has to replace $z^{ \pm} \rightarrow z^{\mp}$ and $x^{ \pm} \rightarrow x^{\mp}$. There are integral representations for $\Phi$ and $\Psi$ as

$$
\begin{gathered}
\Phi\left(x_{1}, x_{2}\right)=i \oint_{C_{1}} \frac{d w_{1}}{2 \pi i} \oint_{C_{1}} \frac{d w_{2}}{2 \pi i} \frac{1}{w_{1}-x_{1}} \frac{1}{w_{2}-x_{2}} \log \frac{\Gamma\left(1+i g\left(w_{1}+w_{1}^{-1}-w_{2}-w_{2}^{-1}\right)\right)}{\Gamma\left(1-i g\left(w_{1}+w_{1}^{-1}-w_{2}-w_{2}^{-1}\right)\right)} \\
\Psi\left(x_{1}, x_{2}\right)=i \oint_{C_{1}} \frac{d w_{2}}{2 \pi i} \frac{1}{w_{2}-x_{2}} \log \frac{\Gamma\left(1+i g\left(x_{1}+x_{1}^{-1}-w_{2}-w_{2}^{-1}\right)\right)}{\Gamma\left(1-i g\left(x_{1}+x_{1}^{-1}-w_{2}-w_{2}^{-1}\right)\right)}
\end{gathered}
$$

where the integrations are for the unit circle. They are well-defined provided none of the $x_{i}$ lies on the unit circle.

As by now we have collected all the necessary formulas we turn to analyze their weak coupling expansions.

\section{Weak coupling expansion}

Our aim is to calculate the weak coupling expansion of $\Delta E$ for $L=1$. In doing so we decompose the integrand of the Lüscher correction (2)

$$
\Delta E=-\sum_{Q=1}^{\infty} \int \frac{d q}{2 \pi} P(q, Q) \Sigma(q, Q)
$$

into a simpler rational part

$$
P(q, Q)=\frac{4 x^{2}\left(1+2 Q z^{-}\left(x-z^{+}\right)\right)^{2}}{\left(\left(x z^{-}\right)^{2}-1\right)\left(x^{2}-\left(z^{+}\right)^{2}\right)}\left(\frac{z^{-}}{z^{+}}\right)^{2}
$$

which contains both the matrix part and the rational part of the scalar factor, and into the more complicated $\Sigma$ part:

$$
\begin{aligned}
i \log \Sigma(q, Q)= & 2\left(\Phi\left(z^{+}, x^{+}\right)-\Phi\left(z^{+}, x^{-}\right)-\Phi\left(z^{-}, x^{+}\right)+\Phi\left(z^{-}, x^{-}\right)\right) \\
& -\Psi\left(z^{+}, x^{+}\right)+\Psi\left(z^{+}, x^{-}\right)-\Psi\left(z^{-}, x^{+}\right)+\Psi\left(z^{-}, x^{-}\right)
\end{aligned}
$$


We expand these functions in $g^{2}$ as

$$
\begin{gathered}
P(q, Q)=P_{8}(q, Q) g^{8}+P_{10}(q, Q) g^{10}+P_{12}(q, Q) g^{12}+\ldots \\
\Sigma(q, Q)=1+\Sigma_{2}(q, Q) g^{2}+\Sigma_{4}(q, Q) g^{4}+\ldots
\end{gathered}
$$

The expansion of the rational part is quite straightforward and we obtain

$$
\begin{gathered}
P_{8}(q, Q)=\frac{4096 Q^{2}\left(-1+q^{2}+Q^{2}\right)^{2}}{\left(q^{2}+Q^{2}\right)^{4}\left(q^{4}+\left(-1+Q^{2}\right)^{2}+2 q^{2}\left(1+Q^{2}\right)\right)} \\
\frac{P_{10}(q, Q)}{P_{8}(q, Q)}=-\frac{8\left(7 q^{4}+3\left(Q^{2}-1\right)^{2}+10 q^{2}\left(1+Q^{2}\right)\right)}{\left(q^{2}+Q^{2}\right)\left(q^{4}+\left(-1+Q^{2}\right)^{2}+2 q^{2}\left(1+Q^{2}\right)\right)} \\
\frac{P_{12}(q, Q)}{P_{10}(q, Q)}=-\frac{2\left(27+268 q^{2}+704 q^{4}\right)}{\left(3+28 q^{2}+64 q^{4}\right)\left(q^{2}+Q^{2}\right)}+\frac{16\left(-3 q^{2}+2 q^{2} Q\right)}{\left(1+4 q^{2}\right)\left(1+q^{2}-2 Q+Q^{2}\right)} \\
-\frac{16\left(3 q^{2}+2 q^{2} Q\right)}{\left(1+4 q^{2}\right)\left(1+q^{2}+2 Q+Q^{2}\right)}-\frac{8\left(-81 q^{2}-117 q^{4}+16 q^{6}+27 q^{2} Q^{2}+16 q^{4} Q^{2}\right)}{\left(3+16 q^{2}\right)\left(3+10 q^{2}+7 q^{4}-6 Q^{2}+10 q^{2} Q^{2}+3 Q^{4}\right)}
\end{gathered}
$$

In expanding the $\Psi$ and $\Phi$ functions we use the same method we used in [7]. The expansion of the $\Psi\left(x_{1}, x_{2}\right)$ functions for $\left|x_{2}\right|>1$ (string region) reads as follows

$$
\begin{aligned}
\Psi\left(x_{1}, x_{2}\right)= & -\frac{g}{x_{2}}\left(\Psi\left(1-i g\left(x_{1}+x_{1}^{-1}\right)\right)+\Psi\left(1+i g\left(x_{1}+x_{1}^{-1}\right)\right)\right) \\
& -\frac{i g^{2}}{2 x_{2}^{2}}\left(\Psi_{1}\left(1-i g\left(x_{1}+x_{1}^{-1}\right)\right)-\Psi_{1}\left(1+i g\left(x_{1}+x_{1}^{-1}\right)\right)\right) \\
& +\frac{g^{3}}{2 x_{2}}\left(\Psi_{2}\left(1-i g\left(x_{1}+x_{1}^{-1}\right)\right)+\Psi_{2}\left(1+i g\left(x_{1}+x_{1}^{-1}\right)\right)\right)+\ldots
\end{aligned}
$$

where $\Psi_{n}(x)=\left(\frac{d}{d x}\right)^{n}\left(\log (\Gamma(x))\right.$ are the standard polygamma functions. If $\left|x_{1}\right|>1$ then $\Phi\left(x_{1}, x_{2}\right)$ starts at $g^{6}$. In the opposite case using the identity $\Phi\left(x_{1}, x_{2}\right)=\Phi\left(0, x_{2}\right)-\Phi\left(x_{1}^{-1}, x_{2}\right)$, being valid if $\left|x_{1}\right| \neq 1$, we can calculate the leading expansion of $\Phi$ as

$$
\Phi(0, x)=\frac{2}{x}\left(\gamma_{E} g-3 \zeta(3) g^{3}+\ldots\right)
$$

Using functional identities valid for integer $Q$ we obtained

$$
\Sigma_{2}(q, Q)=-\frac{16 Q}{q^{2}+Q^{2}}-8\left(S_{1}\left(\frac{Q-i q-2}{2}\right)+S_{1}\left(\frac{Q+i q-2}{2}\right)\right)
$$

and

$$
\begin{aligned}
\Sigma_{4}(q, Q)= & \frac{1}{2} \Sigma_{2}(q, Q)^{2}+\frac{64 Q\left(1+q^{2}+Q^{2}\right)}{\left(q^{2}+Q^{2}\right)^{2}}-\frac{32 i q}{q^{2}+Q^{2}}\left(S_{2}\left(\frac{Q-i q-2}{2}\right)-S_{2}\left(\frac{Q+i q-2}{2}\right)\right)+ \\
& 32\left(S_{1}\left(\frac{Q-i q-2}{2}\right)+S_{1}\left(\frac{Q+i q-2}{2}\right)\right)+8\left(S_{3}\left(\frac{Q-i q-2}{2}\right)+S_{3}\left(\frac{Q+i q-2}{2}\right)\right)+18 \zeta(3)
\end{aligned}
$$

where $S_{n}(x)$ are the analytical continuation of the harmonic sums $S_{n}(N)=\sum_{k=1}^{N} \frac{1}{k^{n}}$. They are related to the polygamma functions as 1

$$
\Psi_{n}(Q)=(-1)^{n+1} n !\left(\zeta(n+1)-S_{n+1}(Q-1)\right.
$$

Let us use now these expressions to calculate the four, five and six loop wrapping corrections.

\footnotetext{
${ }^{1}$ For $n=0$ one has to replace $\zeta(1)$ with $\gamma_{E}$.
} 


\section{Summary of the results}

Once we know the expansion of all functions we can systematically calculate the wrapping corrections by evaluating the expression:

$$
\Delta E=-\sum_{Q=1}^{\infty} \int \frac{d q}{2 \pi}\left[g^{8} P_{8}+g^{10}\left(P_{10}+P_{8} \Sigma_{2}\right)+g^{12}\left(P_{12}+P_{10} \Sigma_{2}+P_{8} \Sigma_{4}\right)+\ldots\right]
$$

Let us analyze the energy correction order by order.

\section{4 loop contribution}

The first non-vanishing term is

$$
\Delta E_{4}=-\sum_{Q=1}^{\infty} \int \frac{d q}{2 \pi} P_{8}(q, Q)
$$

The integrand can be decomposed as

$$
P_{8}(q, Q)=\sum_{j=1}^{4} \frac{R_{8 j}^{-}(Q)}{(q-i Q)^{j}}+\sum_{j=1}^{4} \frac{R_{8 j}^{+}(Q)}{(q+i Q)^{j}}+\frac{R_{8}^{--}(Q)}{q-i Q-i}+\frac{R_{8}^{+-}(Q)}{q+i Q-i}+\frac{R_{8}^{-+}(Q)}{q-i Q+i}+\frac{R_{8}^{++}(Q)}{q+i Q+i}
$$

This form is actually quite generic, that is we can always expand the rational part of the integrand as

$$
P_{2 k}(q, Q)=\sum_{\epsilon= \pm} \sum_{j=1}^{2 k} \frac{R_{2 k j}^{\epsilon}(Q)}{(q+\epsilon i Q)^{j}}+\sum_{\epsilon_{1}, \epsilon_{2}=\{ \pm\}} \sum_{j=1}^{k} \frac{R_{2 k j}^{\epsilon_{1} \epsilon_{2}}(Q)}{\left(q+\epsilon_{1} i Q+\epsilon_{2} i\right)^{j}}
$$

As the function $P_{8}$ is real $R_{8 j}^{-}(Q)^{*}=R_{8 j}^{+}(Q)$ and $R_{8}^{--}(Q)^{*}=R_{8}^{++}(Q), R_{8}^{+-}(Q)^{*}=R_{8}^{-+}(Q)$. The various coefficients can be expressed in negative powers of $Q$ and $2 Q \pm 1$ as

$$
\begin{gathered}
R_{84}^{+}(Q)=\frac{256}{Q^{2}}-\frac{512}{(2 Q-1)}+\frac{512}{(2 Q+1)} \quad ; \quad R_{83}^{+}(Q)=\frac{512 i}{Q^{3}}-\frac{512 i}{(2 Q-1)^{2}}+\frac{512 i}{(2 Q+1)^{2}} \\
R_{82}^{+}(Q)=-\frac{640}{Q^{4}}+\frac{512}{Q^{2}}+\frac{512}{(2 Q-1)^{3}}-\frac{512}{(2 Q-1)}-\frac{512}{(2 Q+1)^{3}}+\frac{512}{(2 Q+1)} \\
R_{81}^{+}(Q)=-\frac{640 i}{Q^{5}}+\frac{512 i}{Q^{3}}+\frac{512 i}{(2 Q-1)^{4}}-\frac{512 i}{(2 Q-1)^{2}}-\frac{512 i}{(2 Q+1)^{4}}+\frac{512 i}{(2 Q+1)^{2}} \\
R_{8}^{--}(Q)=-\frac{512 i}{(2 Q+1)^{4}}+\frac{512 i}{(2 Q+1)^{2}} ; \quad R_{8}^{-+}(Q)=\frac{512 i}{(2 Q-1)^{4}}-\frac{512 i}{(2 Q+1)^{2}}
\end{gathered}
$$

We can further decompose any function as

$$
R_{8 j}^{ \pm}=R_{8 j, Q}^{ \pm}+R_{8 j, 2 Q+1}^{ \pm}+R_{8 j, 2 Q-1}^{ \pm} \quad ; \quad R_{8 j, Q}^{ \pm}=\sum_{k=1}^{5} \frac{R_{8 j, Q k}^{ \pm}}{Q^{k}} \quad ; \quad R_{8 j,(2 Q \pm 1)}^{ \pm}=\sum_{k=1}^{4} \frac{R_{8 j,(2 Q \pm 1) k}^{ \pm}}{(2 Q \pm 1)^{k}}
$$

(It can be extended an analogous way for any $R_{2 k j}$ ). We calculate the integral by residues in which we take into account the poles on the upper half plane. They are located at $q=i Q$ which we call the kinematical pole and at $q=i(Q \pm 1)$ which are the dynamical poles. The contributions of the dynamical poles are canceled when summed over the bound-state contributions $(Q)$. Thus we have to keep only the pole at $i Q$ which result in the following sum

$$
\Delta E_{4}=-\sum_{Q=1}^{\infty} \operatorname{Res}_{q=i Q} P_{8}(q, Q)=-\sum_{Q=1}^{\infty} R_{81}^{-}(Q)=128(4 \zeta(3)-5 \zeta(5))
$$


In summing up the $R_{81,2 Q \pm 1}$ terms here and later on we use the following trick:

$$
\sum_{Q=1}^{\infty}\left(R_{8 j, 2 Q-1}^{ \pm}(Q)+R_{8 j, 2 Q+1}^{ \pm}(Q)\right)=R_{8 j, 2 Q-1}^{ \pm}(1)+\sum_{Q=1}^{\infty}\left(R_{8 j, 2 Q-1}^{ \pm}(Q+1)+R_{8 j, 2 Q+1}^{ \pm}(Q)\right)=0
$$

This means that in the sum only the $Q^{-n}$ type terms survive, which result in the sum $\sum_{Q=1}^{\infty} Q^{-n}$ and contains single zeta functions reproducing the result of [6].

\section{5 loop contribution}

The second order correction has the form

$$
\Delta E_{5}=-\sum_{Q=1}^{\infty} \int \frac{d q}{2 \pi}\left(P_{10}(q, Q)+P_{8}(q, Q) \Sigma_{2}(q, Q)\right)
$$

Similarly to the 4 loop result in the first term only the residue at $i Q$ contributes and we obtain a single sum: $P_{10}$ has a similar decomposition as $P_{8}$ and only the $\sum_{Q=1}^{\infty} Q^{-n}=\zeta(n)$ terms survive which results in

$$
-\sum_{Q=1}^{\infty} \int \frac{d q}{2 \pi} P_{10}(q, Q)=-\sum_{Q=1}^{\infty} R_{101, Q}^{-}(Q)=-128(48 \zeta(3)+40 \zeta(5)-105 \zeta(7))
$$

In calculating the integral of the term $P_{8}(q, Q) \Sigma_{2}(q, Q)$ we can use the fact that $P_{8}$ is real and replace in $\Sigma_{2}$ the expression $S_{1}\left(-1+\frac{Q+i q}{2}\right)$ with its complex conjugate $S_{1}\left(-1+\frac{Q-i q}{2}\right)$ :

$$
\tilde{\Sigma}_{2}(q, Q)=-\frac{16 Q}{q^{2}+Q^{2}}-16 S_{1}\left(\frac{Q-i q-2}{2}\right)
$$

and take the real part of the integral. Interestingly the integral is real in this case and we have

$$
\int \frac{d q}{2 \pi}\left(P_{8}(q, Q) \Sigma_{2}(q, Q)\right)=\int \frac{d q}{2 \pi}\left(P_{8}(q, Q) \tilde{\Sigma}_{2}(q, Q)\right)=\Delta E_{5 Q}+\Delta E_{5(2 Q \pm 1)}
$$

The replacement has the advantage that $S_{1}\left(-1+\frac{Q-i q}{2}\right)$ does not have any pole on the upper half plane opposed to $S_{1}\left(-1+\frac{Q+i q}{2}\right)$. Thus calculating the integral we can take the residues at $i Q$ and $i(Q \pm 1)$ only. We use the same method in calculating the sums for the $2 Q \pm 1$ type terms as we used for $\Delta_{4}$ : we evaluate the $Q=1$ term in the $(2 Q-1)$ type terms, then we add the $Q \rightarrow Q+1$ shifted terms to the $2 Q+1$ type terms. These terms include the dynamical residues and the $R_{8 j(2 Q \pm 1)}^{-}$type kinematical residue and result in

$$
\Delta E_{5(2 Q \pm 1)}=\frac{256}{27} \pi^{4}\left(\pi^{2}-6\right)+2048 \zeta(3)
$$

- From the remaining $R_{8 j Q}$ type terms at the kinematical residue we got summands of the form $\sum_{Q=1}^{\infty} Q^{-n} S_{m}(Q-1)=\zeta(n, m)$, for $n+m=4$ and $n+m=6$. Explicitly summing them up we obtained

$\Delta E_{5 Q}=512\left(8 \zeta(2)^{2}-4 \zeta(3)^{2}-11 \zeta(2) \zeta(4)-8 \zeta(2,2)+\zeta(2,4)-16 \zeta(3,1)+4 \zeta(3,3)+10 \zeta(4,2)+20 \zeta(5,1)\right)$

We can express the multiple zeta values in terms of products of simple zeta functions either by the online high precision calculator of MZVs: EZFACE, or analytically, by using the algebraic relations between them [33, 34] and the data mine for MZVs: 28]. Combining all contributions we arrive at the final 5 loop formula

$$
\Delta E_{5}=-128\left(12 \zeta(3)^{2}+32 \zeta(3)+40 \zeta(5)-105 \zeta(7)\right)
$$




\section{6 loop contribution}

The calculation of the six loop formula reduces to the evaluation of

$$
\Delta E_{6}=-\sum_{Q=1}^{\infty} \int \frac{d q}{2 \pi}\left(P_{12}(q, Q)+P_{10}(q, Q) \Sigma_{2}(q, Q)+P_{8}(q, Q) \Sigma_{4}(q, Q)\right)
$$

The various terms have different structure in terms of multiple zeta values. The first term can be evaluated just by taking the residue at $i Q$ and summing up the $\sum_{Q=1}^{\infty} Q^{-n}=\zeta(n)$ type single zeta function contributions of $R_{121, Q}^{-}(Q)$ :

$$
128(576 \zeta(3)+480 \zeta(5)+392 \zeta(7)-1701 \zeta(9))
$$

In evaluating the term $P_{10} \Sigma_{2}$ we use the trick to replace $S_{1}\left(-1+\frac{Q+i q}{2}\right)$ with its complex conjugate $S_{1}\left(-1+\frac{Q-i q}{2}\right)$ and calculate the integral of $P_{10} \tilde{\Sigma}_{2}$ by taking into account the contributions of the residues at $i Q$ and $i(Q \pm 1)$ only. The results can be decomposed into the contributions of the dynamical poles together with the $R_{10 j(2 Q \pm 1)}^{-}$type terms of the kinematical pole and into the most complicated contribution of the kinematical pole $R_{10 j Q}^{-}$terms. This latter one can be written in terms of MZVs as $\sum_{Q=1}^{\infty} Q^{-n} S_{m}(Q-1)=\zeta(n, m)$, for $n+m=4,6,8$. Explicitly summing up this term we obtain

$$
\begin{array}{r}
-1024\left(48 \zeta(2)^{2}+(16-15 \zeta(4)) \zeta(4)+8 \zeta(3)(\zeta(3)-6 \zeta(5))+\zeta(2)(32 \zeta(4)-105 \zeta(6))-8 \zeta(6)\right. \\
-48 \zeta(2,2)-80 \zeta(3,1)+8 \zeta(2,4)-8 \zeta(3,3)-40 \zeta(4,2)-88 \zeta(5,1) \\
+15 \zeta(4,4)+3 \zeta(3,5)+45 \zeta(5,3)+105 \zeta(6,2)+210 \zeta(7,1))
\end{array}
$$

The basis of the multiple zeta values contain an irreducible depth two element which can be chosen to be $\zeta(3,5)$, see [28. Interestingly, however, using the relations between the MZVs, 28], we could express the total contribution in terms of products of single zeta values as

$$
\frac{512}{675}\left(-1200 \pi^{4}-80 \pi^{6}+27 \pi^{8}+60750 \zeta(3) \zeta(5)\right)
$$

The other remaining terms give single zeta functions only. There are two terms

$$
131072 \frac{Q(1+Q)}{(1+2 Q)^{4}} \Psi_{1}(Q)-65536 \frac{Q(1+Q)}{(1+2 Q)^{3}} \Psi_{2}(Q)-\frac{4096}{(1+2 Q)^{2}} \Psi_{3}(Q)+\frac{2048}{3(1+2 Q)} \Psi_{4}(Q)
$$

and

$$
-131072 \frac{Q(1+Q)}{(1+2 Q)^{4}} \Psi_{1}\left(Q+\frac{1}{2}\right)
$$

whose summation leads to alternating Euler sums. We do not write them here as these terms will cancel when we combine them with other terms coming from $P_{8} \Sigma_{4}$.

In calculating $P_{8} \Sigma_{4}$, the trick of replacing $S_{n}\left(-1+\frac{Q+i q}{2}\right)$ with its complex conjugate $S_{n}\left(-1+\frac{Q-i q}{2}\right)$ and taking the real part of the result can be applied. This works equally for $S_{1}\left(-1+\frac{Q \pm i q}{2}\right) S_{1}(-1+$ $\left.\frac{Q \pm i q}{2}\right)$, but not for $S_{1}\left(-1+\frac{Q+i q}{2}\right) S_{1}\left(-1+\frac{Q-i q}{2}\right)$ which is manifestly real and needs a special care. For this reason, we separate $\Sigma_{4}$ as

$$
\Sigma_{4}=\Re \mathrm{e}\left(\tilde{\Sigma}_{4}\right)+64 S_{1}\left(-1+\frac{Q+i q}{2}\right) S_{1}\left(-1+\frac{Q-i q}{2}\right)
$$

where in $\tilde{\Sigma}_{4}$ the replacement can be applied and it contains the rational part, too. The advantage of using $\tilde{\Sigma}_{4}$ is that it does not have any pole on the upper half plane, so in calculating its contributions only the kinematical $(i Q)$ an dynamical poles $(i(Q \pm 1))$ have to be taken into account. In contrast, additionally to these poles, $S_{1}\left(-1+\frac{Q+i q}{2}\right)$ has simple poles at $q=i(2 n+Q)$ with residue 2 for any 
integers $n$. Taking into account the additional pole contributions we encounter a double sum of the form:

$$
\sum_{Q=1}^{\infty} \sum_{n=1}^{\infty} 128 P_{8}(i(2 n+Q), Q) S_{1}(n+Q-1)
$$

We first separate the contributions coming from $R_{8 j, Q}^{ \pm}, R_{8 j, 2 Q \pm 1}^{ \pm}$and $R_{8}^{ \pm \pm}, R_{8}^{\mp \pm}$. In calculating the $R_{8 j, Q}^{ \pm}$part we use the result from the Appendix to arrive at:

$$
\begin{array}{r}
2048(6 \zeta(3) \zeta(4)+21 \zeta(4) \zeta(2,1)-\zeta(3) \zeta(2,2)+8 \zeta(2,3)-\zeta(2,5)-4 \zeta(3) \zeta(3,1)+16 \zeta(3,2) \\
-4 \zeta(3,4)+24 \zeta(4,1)+\zeta(2)(8 \zeta(3)-19 \zeta(5)-24 \zeta(2,1)+\zeta(2,3)+4 \zeta(3,2)+12 \zeta(4,1))-10 \zeta(4,3) \\
-20 \zeta(5,2)-35 \zeta(6,1)+16 \zeta(2,2,1)-2 \zeta(2,4,1)+32 \zeta(3,1,1)-8 \zeta(3,3,1)-20 \zeta(4,2,1)-40 \zeta(5,1,1)
\end{array}
$$

In calculating the contributions of $R_{8 j, 2 Q \pm 1}^{ \pm}$we use the usual trick to shift the $2 Q-1$ type terms and combine them with the $2 Q+1$ type terms, but additionally we combine this step with an appropriate shift $n \rightarrow n+1$, too. The resulting expression, apart form a rational part, contains $\Psi(Q+1)$ with a quite long prefactor which we do not write out explicitly here, as it will nicely combine together with the contributions of the kinematical poles. The contributions of $R_{8}^{ \pm \pm}, R_{8}^{\mp \pm}$ can be evaluated along the same lines and we obtain

$$
\frac{262144 Q(1+Q)}{(1+2 Q)^{4}} \Psi\left(\frac{3}{2}+Q\right)
$$

Finally we have to calculate the contributions of the kinematical and dynamical poles. The dynamical poles are simple and result in poly gamma functions of the form $\Psi_{i}\left(\frac{1}{2}+Q\right)$ for $i=0,1$. Combining their contributions with (89) we obtain the simple result:

$$
4096(28 \zeta(3)-31 \zeta(5))
$$

The calculation of the contributions of the kinematical pole $(q=i Q)$ is the most complicated. It slightly simplifies when we collect the remaining contributions from (7) and from the term coming from the $\Psi(Q+1)$ type term of the residues at $i(Q+2 n)$. The emerging sums, apart from rational expressions which can summed up easily with Mathematica, contain the products of harmonic numbers multiplied with rational functions. Decomposing them we have terms of the form

$$
\sum_{Q=1}^{\infty} \frac{1}{Q^{k}} S_{n}(Q-1) S_{m}(Q-1)
$$

for $m+n=5,6,7$ or 8 and alternating sums of the form

$$
\sum_{Q=1}^{\infty}\left(\frac{1}{(-1+2 Q)^{k}}-\frac{1}{(1+2 Q)^{k}}\right) S_{n}(Q-1) S_{m}(Q-1)
$$

for $n, m$ and $k \leq 5$. The latter one can be treated by the usual trick of shifting $Q$ in the first term, and evaluating the $Q=1$ term separately. The difference of the resulting shifted harmonic sums can be simplified into a single harmonic sum, which then can be evaluated in terms of MZVs of depth two containing also individual irreducible elements like $\zeta(3,5)$. In the expressions (10) we can use that

$$
S_{n}(Q-1) S_{m}(Q-1)=\sum_{j=1}^{Q-1} \frac{1}{j^{n}} \sum_{k=1}^{j-1} \frac{1}{k^{m}}+\sum_{j=1}^{Q-1} \frac{1}{j^{m}} \sum_{k=1}^{j-1} \frac{1}{k^{n}}+S_{n+m}(Q-1)
$$

This will lead to multivariate zeta functions of the form $\xi(k, n, m), \xi(k, m, n)$ and $\zeta(k, m+n)$. Unfortunately the emerging expressions are not very illuminating and are rather lengthy. Magically, however, 
when we combine all the contributions the irreducible $\zeta(3,5)$ term disappears and we arrive at the nice result of the 6 loop correction which is:

$$
\Delta E_{6}=-128\left(48 \zeta(3)^{2}-592 \zeta(5)-24 \zeta(3)(8+15 \zeta(5))-322 \zeta(7)+1701 \zeta(9)\right)
$$

In structure it is very similar to the result of other six loop calculations, like the NLO result for the twist three [29] or single impurity 23] operators. Let us finally mention, that we evaluated numerically the integral form of $\Delta E_{6}$ and the result perfectly agreed with the exact analytical result (11).

\section{Conclusion}

In the paper we have evaluated Lüscher's finite size energy formula for the simplest case in the AdS/CFT correspondence at NNLO and determined the anomalous dimension of a single impurity operator in the $\beta=\frac{1}{2}$ deformed theory at six loops. The Lüscher formula expresses the energy correction in terms of the scattering matrix of the physical particle and the mirror bound-states. In calculating the weak coupling expansion of the correction we have to integrate the expanded scattering matrix elements for the mirror momentum and sum over the full bound-state spectrum $Q \in \mathbb{Z}$. The expansion of the dressing phase in the mirror-physical kinematics at order $n$ contains the $(n-1)^{t h}$ power of the polygamma function. When integrated over the mirror momentum by residues derivatives of polygamma functions evaluated at integer values appear. These can be expressed in terms of nested harmonic sums which after summation over the bound-state spectrum result in multiple zeta values. At the $n^{\text {th }}$ order of the expansion MZVs of depth $n$ appear, additionally, the degree of the MZV is increased by 2 from order to order. Irreducible MZVs, those which cannot be written in terms of products of zeta functions appear already at depth 2 and degree 8. Although during the calculation of the six loop anomalous dimension of the single impurity operator irreducible MZVs appeared, but in the final form they canceled out, leaving only products of zeta functions.

We have no explanation why such a cancellation appeared and it would nice to evaluate the seven loop correction in this setting to see whether a similar effect takes place at the next order, too. It would be even more interesting to calculate the eight loop correction, since there a new type of double wrapping effect will appear. Another interesting problem is to evaluate these corrections using Laplace transformations [7, as in this case integrals of polylogarithms appear. Then using the theory of motives just as for scattering amplitudes in 35 might explain the form of the correction.

The developed techniques can be extended to calculate the six loop anomalous dimension of the Konishi operator, which corresponds to a two particle state on the string side. We expect that the form of the wrapping correction will be the same, only the actual coefficients will change. This result would be very relevant to check to proposed excited TBA equations [17, 18]. Indeed, the comparison of the infinite coupled TBA equation to the Lüscher correction at five loops [24, 25, 23] confirmed only part of the TBA equations, while a six loop analysis would check the full set.

The form of the correction we obtained might shed some light on how a possible finite system of NLIE type integral equations would look like as the weak coupling expansion of the anomalous dimension is very similar in structure to the expansion of the BES equation [36].

\section{Acknowledgments}

We thank Romuald Janik and Tomek Lukowski for the useful discussions, for Matthias Staudacher to calling our attention to MZVs and for Johannes Blümlein to reminding us the MZVs data mine. The work was supported by a Bolyai Scholarship, and by OTKA K81461.

\section{A Summing up nested harmonic sums}

Here in this appendix we describe how we summed up nested harmonic sums. 
At 6 loops we have triple and double sums and here we focus on the triple sums only. Consider a sum of the form

$$
f(a, b)=\sum_{Q=1}^{\infty} \frac{1}{Q^{a}} \sum_{n=1}^{\infty} \frac{1}{n^{b}} S_{1}(n+Q-1)
$$

We claim that the result is

$$
\begin{aligned}
f(a, b)= & \zeta(a) \zeta(b, 1)+\zeta(a) \zeta(b+1) \\
& +\sum_{j=0}^{b-2}(-1)^{j} \zeta(b-j) \zeta(a, j+1)-(-1)^{b}(\zeta(a, b+1)+\zeta(a, b, 1))
\end{aligned}
$$

where the Euler-Zagier sums are defined as

$$
\zeta\left(a_{1}, a_{2}, \ldots, a_{n}\right)=\sum_{j_{1}>j_{2}>\ldots>j_{n}>0} \frac{1}{j_{1}^{a_{1}} j_{2}^{a_{2}} \ldots j_{n}^{a_{n}}}
$$

The derivation goes as follows:

First we use that $S_{1}(n+Q-1)=\sum_{j=1}^{n+Q-1} \frac{1}{j}$ and decompose the sum as

$$
\sum_{Q=1}^{\infty} \frac{1}{Q^{a}} \sum_{n=1}^{\infty} \frac{1}{n^{b}}\left(\sum_{j=1}^{n-1} \frac{1}{j}+\frac{1}{n}+\sum_{j=1}^{Q-1} \frac{1}{n+j}\right)=\zeta(a) \zeta(b, 1)+\zeta(a) \zeta(b+1)+\sum_{Q=1}^{\infty} \frac{1}{Q^{a}}\left(\sum_{n=1}^{\infty} \frac{1}{n^{b}} \sum_{j=1}^{Q-1} \frac{1}{n+j}\right)
$$

Now exchanging the orders of the sums and using that

$$
\sum_{j=1}^{Q-1}\left(\sum_{n=1}^{\infty} \frac{1}{n^{b}} \frac{1}{n+j}\right)=\sum_{j=1}^{Q-1}\left(\sum_{k=0}^{b-2} \frac{(-1)^{k}}{j^{k+1}} \zeta(b-k)-\frac{(-1)^{b}}{j^{b}}\left(\frac{1}{j}+\sum_{k=1}^{j-1} \frac{1}{k}\right)\right)
$$

we can write

$$
\sum_{Q=1}^{\infty} \frac{1}{Q^{a}}\left(\sum_{n=1}^{\infty} \frac{1}{n^{b}} \sum_{j=1}^{Q-1} \frac{1}{n+j}\right)=\sum_{k=0}^{b-2}(-1)^{k} \zeta(a, k+1) \zeta(b-k)-(-1)^{b}(\zeta(a) \zeta(b+1)+\zeta(a, b, 1))
$$

An easier manipulation gives

$$
\sum_{Q=1}^{\infty} \frac{1}{Q^{a}} \sum_{n=1}^{\infty} \frac{1}{(n+Q)^{b}} S_{1}(n+Q-1)=\zeta(a) \zeta(b, 1)-\zeta(a+b, 1)-\zeta(a, b, 1)
$$

In the 6 loop calculation terms with $b=1$ appear. They are individually divergent but their appearing combination is finite.

\section{References}

[1] J. M. Maldacena, The large N limit of superconformal field theories and supergravity, Adv. Theor. Math. Phys. 2 (1998) 231-252. arXiv:hep-th/9711200.

[2] G. Arutyunov, S. Frolov, Foundations of the AdS5 x S5 Superstring. Part I, J. Phys. A42 (2009) 254003. arXiv:0901.4937, doi:10.1088/1751-8113/42/25/254003.

[3] Z. Bajnok, R. A. Janik, Four-loop perturbative Konishi from strings and finite size effects for multiparticle states, Nucl. Phys. B807 (2009) 625-650. arXiv:0807.0399, doi:10.1016/j.nuclphysb.2008.08.020 
[4] F. Fiamberti, A. Santambrogio, C. Sieg, D. Zanon, Wrapping at four loops in N=4 SYM, Phys. Lett. B666 (2008) 100-105. arXiv:0712.3522, doi:10.1016/j.physletb.2008.06.061.

[5] F. Fiamberti, A. Santambrogio, C. Sieg, D. Zanon, Anomalous dimension with wrapping at four loops in N=4 SYM, Nucl. Phys. B805 (2008) 231-266. arXiv:0806.2095, doi:10.1016/j.nuclphysb.2008.07.014

[6] Z. Bajnok, R. A. Janik, T. Lukowski, Four loop twist two, BFKL, wrapping and strings, Nucl. Phys. B816 (2009) 376-398. arXiv:0811.4448, doi:10.1016/j.nuclphysb.2009.02.005

[7] Z. Bajnok, A. Hegedus, R. A. Janik, T. Lukowski, Five loop Konishi from AdS/CFTarXiv:0906.4062.

[8] A. Mauri, S. Penati, A. Santambrogio, D. Zanon, Exact results in planar N = 1 superconformal Yang-Mills theory, JHEP 11 (2005) 024. arXiv:hep-th/0507282.

[9] F. Fiamberti, A. Santambrogio, C. Sieg, D. Zanon, Finite-size effects in the superconformal beta-deformed $\mathrm{N}=4$ SYM, JHEP 08 (2008) $057 . \quad$ arXiv:0806.2103, doi:10.1088/1126-6708/2008/08/057.

[10] F. Fiamberti, A. Santambrogio, C. Sieg, D. Zanon, Single impurity operators at critical wrapping order in the beta-deformed $\mathrm{N}=4$ SYM, JHEP 08 (2009) 034. arXiv:0811.4594, doi:10.1088/1126-6708/2009/08/034

[11] N. Beisert, R. Roiban, Beauty and the twist: The Bethe ansatz for twisted N $=4$ SYM, JHEP 08 (2005) 039. arXiv:hep-th/0505187.

[12] C. Ahn, Z. Bajnok, D. Bombardelli, R. I. Nepomechie, Finite-size effect for four-loop Konishi of the beta- deformed $\mathrm{N}=4 \mathrm{SYM}$, arXiv:1006.2209

[13] N. Gromov, V. Kazakov, P. Vieira, Exact Spectrum of Anomalous Dimensions of Planar N=4 Supersymmetric Yang-Mills Theory, Phys. Rev. Lett. 103 (2009) 131601. arXiv:0901.3753, doi:10.1103/PhysRevLett.103.131601.

[14] G. Arutyunov, S. Frolov, String hypothesis for the $A d S_{5} \times S^{5}$ mirror, JHEP 03 (2009) 152 . arXiv:0901.1417, doi:10.1088/1126-6708/2009/03/152.

[15] D. Bombardelli, D. Fioravanti, R. Tateo, Thermodynamic Bethe Ansatz for planar AdS/CFT: a proposal, J. Phys. A42 (2009) 375401. arXiv:0902.3930, doi:10.1088/1751-8113/42/37/375401.

[16] G. Arutyunov, S. Frolov, Thermodynamic Bethe Ansatz for the $A d S_{5} x S^{5}$ Mirror Model, JHEP 05 (2009) 068. arXiv:0903.0141, doi:10.1088/1126-6708/2009/05/068.

[17] N. Gromov, V. Kazakov, A. Kozak, P. Vieira, Exact Spectrum of Anomalous Dimensions of Planar $\mathrm{N}=4$ Supersymmetric Yang-Mills Theory: TBA and excited states, Lett. Math. Phys. 91 (2010) 265-287. arXiv:0902.4458, doi:10.1007/s11005-010-0374-8

[18] G. Arutyunov, S. Frolov, R. Suzuki, Exploring the mirror TBA, JHEP 05 (2010) 031. arXiv:0911.2224, doi:10.1007/JHEP05(2010) 031 .

[19] A. Cavaglia, D. Fioravanti, R. Tateo, Extended Y-system for the $A d S_{5} / C F T_{4}$ correspondence, arXiv:1005.3016.

[20] N. Gromov, F. Levkovich-Maslyuk, Y-system and beta-deformed N=4 Super-Yang-Mills, arXiv: 1006.5438 
[21] C. Ahn, Z. Bajnok, D. Bombardelli, R. I. Nepomechie, Twisted Bethe equations from a twisted S-matrix, arXiv:1010.3229.

[22] J. Gunnesson, Wrapping in maximally supersymmetric and marginally deformed $\mathrm{N}=4$ Yang-Mills, JHEP 04 (2009) 130. arXiv:0902.1427, doi:10.1088/1126-6708/2009/04/130

[23] G. Arutyunov, M. de Leeuw, S. J. van Tongeren, Twisting the Mirror TBA, arXiv:1009.4118,

[24] G. Arutyunov, S. Frolov, R. Suzuki, Five-loop Konishi from the Mirror TBA, JHEP 04 (2010) 069. arXiv:1002.1711, doi:10.1007/JHEP04(2010)069.

[25] J. Balog, A. Hegedus, 5-loop Konishi from linearized TBA and the XXX magnet, JHEP 06 (2010) 080. arXiv:1002.4142, doi:10.1007/JHEP06(2010) 080 .

[26] J. Balog, A. Hegedus, The Bajnok-Janik formula and wrapping corrections, arXiv:1003.4303.

[27] D. H. Bailey, J. M. Borwein, D. Broadhurst, W. Zudilin, Experimental Mathematics and Mathematical Physics, arXiv:1005.0414

[28] J. Blumlein, D. J. Broadhurst, J. A. M. Vermaseren, The Multiple Zeta Value Data Mine, Comput. Phys. Commun. 181 (2010) 582-625. arXiv:0907.2557, doi:10.1016/j.cpc.2009.11.007.

[29] V. N. Velizhanin, Six-Loop Anomalous Dimension of Twist-Three Operators in N=4 SYM, arXiv:1003.4717.

[30] G. Arutyunov, S. Frolov, The S-matrix of String Bound States, Nucl. Phys. B804 (2008) 90-143. arXiv:0803.4323, doi:10.1016/j.nuclphysb.2008.06.005

[31] T. Lukowski, A. Rej, V. N. Velizhanin, Five-Loop Anomalous Dimension of Twist-Two Operators, arXiv:0912.1624

[32] G. Arutyunov, S. Frolov, The Dressing Factor and Crossing Equations, J. Phys. A42 (2009) 425401. arXiv:0904.4575, doi:10.1088/1751-8113/42/42/425401

[33] J. Blumlein, S. Kurth, Harmonic sums and Mellin transforms up to two-loop order, Phys. Rev. D60 (1999) 014018. arXiv:hep-ph/9810241, doi:10.1103/PhysRevD.60.014018.

[34] J. Blumlein, Algebraic relations between harmonic sums and associated quantities, Comput. Phys. Commun. 159 (2004) 19-54. arXiv:hep-ph/0311046, doi:10.1016/j.cpc.2003.12.004.

[35] A. B. Goncharov, M. Spradlin, C. Vergu, A. Volovich, Classical Polylogarithms for Amplitudes and Wilson Loops,arXiv:1006.5703.

[36] N. Beisert, B. Eden, M. Staudacher, Transcendentality and crossing, J. Stat. Mech. 0701 (2007) P021. arXiv: hep-th/0610251. 